\title{
Impact of Scattering in Quasi-Ordered Structures on THz Imaging
}

\author{
I. N. Dolganova $^{1,2,3}$, N. V. Chernomyrdin ${ }^{1,2,4}$, A. A. Kuznetsov ${ }^{1}$, K. M. Malakhov ${ }^{1}$, \\ V. E. Karasik ${ }^{1}$, K. I. Zaytsev ${ }^{1,2,4}$ \\ ${ }^{1}$ Bauman Moscow State Technical University, Moscow, Russia \\ ${ }^{2}$ Sechenov First Moscow State Medical University, Moscow, Russia \\ ${ }^{3}$ Institute of Solid State Physics of the Russian Academy of Sciences, Chernogolovka, Russia \\ ${ }^{4}$ Prokhorov General Physics Institute of RAS, Moscow, Russia \\ in.dolganova@gmail.com
}

Terahertz science and technologies demonstrate a wide range of imaging applications in physics, biology, medicine, industry, etc. [1-7]. Scattering has one of the most essential impacts on imaging quality [14]. The mostly applied methods for estimation of scattering parameters and imaging losses are analytical diffuse and small-angle approximation of radiative transfer theory, Monte Carlo (MC), discrete ordinates, and T-matrix numerical methods, finite-difference time-domain method (FDTD), and experimental methods, e.g., using integrating sphere [8-11].

Nevertheless, one should pay attention to local ordering of scatterers in the considered media, since it could lead to significant changes of scattering signal [1-2].

We have studied the impact of quasi-ordered scattering materials on $\mathrm{THz}$ imaging, using a combined computational approach, based on computational electrodynamics, radiative scattering theory and Monte Carlo simulations. We assumed that the scattering effects could be accounted for by finding a single scattering phase function for clusters of particles, which form quasi-ordered scattering material, and using it in MC simulation of scattering, considering the whole medium as a random structure of such clusters. We applied FDTD technique for solution of Maxwell's equations in the near-field (NF) zone with the NF to far-field (FF) transformation based on the calculation of the diffraction integral. It helped us to determine scattering phase function and include it in MC numerical simulations in order to find scattered radiance $\mathrm{L}(\theta)$ and the corresponding imaging modulation transfer functions (MTF).

The described approach was used to estimate an impact of quasi-ordered media [7,12-13].

We estimated imaging MTF in case of clothes scattering material. The numerical simulations and experimental measurements were performed for the following system parameters: imaging frequency of the $\mathrm{THz}$ source (backward-wave oscillator) was $0.25 \mathrm{THz}$, distance between the imaging system and the bar-pattern test-object was $4 \mathrm{~m}$; for the scattering materials with groups of four cylindrical particles with period $0.3 \mathrm{~mm}$ dielectric permittivity of a single particle of diameter $0.2 \mathrm{~mm}$ was 2.9 ; for the scattering materials with groups of seven cylindrical particles with period $1.34 \mathrm{~mm}$ dielectric permittivity of a single particle of diameter $1.34 \mathrm{~mm}$ was 1.6; the thickness of scattering layers was $0.7 \mathrm{~mm}$ in the first case, and $4 \mathrm{~mm}$ in the second. The experimental scheme and the results are demonstrated in Fig. 1.

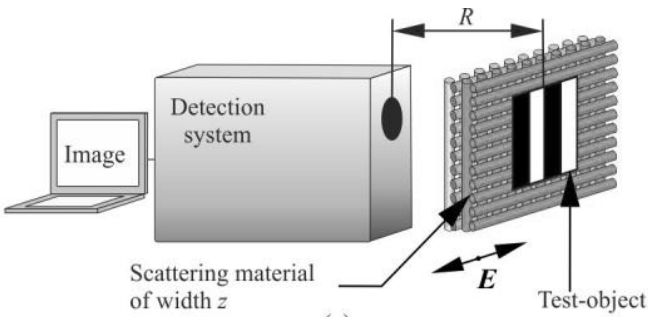

(a)

\begin{tabular}{c|c|c|c|c|c}
\hline \multirow{2}{*}{$\begin{array}{c}\text { Scattering } \\
\text { volume } \\
\text { structure }\end{array}$} & \multicolumn{2}{|c|}{ Random } & \multicolumn{2}{c|}{ Quasi-ordered } & $\begin{array}{c}\text { Diffraction } \\
\text { limited } \\
\text { imaging } \\
\text { system }\end{array}$ \\
\cline { 2 - 5 } & 0 & $\bullet$ & 88 & 88 & - \\
\hline Simulations & - & ---- & & - & - \\
\hline Experiment & $\ldots$ & $\ldots$ & $\bullet$ & $\circ$ & $\bullet$ \\
\hline
\end{tabular}

(b)

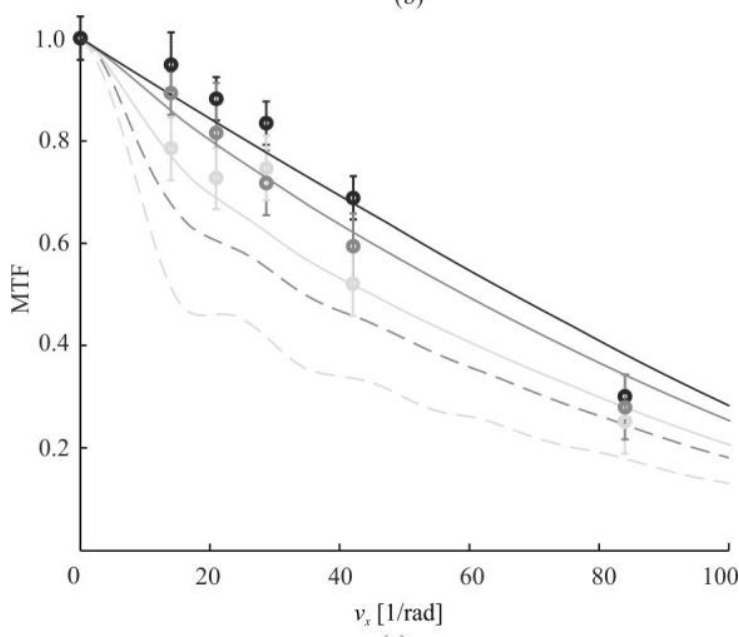

(c)

Fig. 1. (a) An experimental scheme for finding MTF using a set of test-objects; (c) and (d) experimental and numerical results, where black color corresponds to measurement without scattering layer.

We also calculated the MTF in case of random distribution of particles in the scattering materials and in case of quasi-ordering.

According to the numerical simulations [7], it is possible to determine particular combination of wavelength, particle system parameters, local period and dielectric parameters, for which imaging contrast of a remote object (and the corresponding MTF) would increase. On the contrary, almost opaque media could be achieved. 
The demonstrated analysis could be effectively used for finding optimal wavelengths for imaging applications.

This work is supported by the Russian Science Foundation (RSF), Project \# 14-19-01083.

\section{References}

1. Fokina, I. N. et al. Impact of structure geometry on scattering in partially-ordered media // Journal of Quantitative Spectroscopy \& Radiative Transfer 2014. V. 149, P. 108-116.

2. Dolganova, I. N. et al. Combined terahertz imaging system for enhanced imaging quality // Optical and Quantum Electronics 2016. V. 48, No. 6, P. 325.

3. Dolganova, I. N. et al. A hybrid continuous-wave terahertz imaging system // Review of Scientic Instruments 2016. V. 86, P. 113704

4. Chernomyrdin, N. V. et al. Reflection-mode continuous-wave 0.15lambda-resolution terahertz solid immersion microscopy of soft biological tissues // Applied Physics Letters 2018. V. 113, No. 11, P. 111102.

5. Chernomyrdin, N. V. et al. Solid immersion terahertz imaging with sub-

wavelength resolution // Applied Physics Letters 2017. V. 110 , No. 22, P. 221109.

6. Yakovlev, E. V. et al. Non-Destructive Evaluation of Polymer Composite Materials at the Manufacturing Stage
Using Terahertz Pulsed Spectroscopy // IEEE Transactions on Terahertz Science and Technology 2015. V. 5, No. 5, P. 810-816.

7. Dolganova, I. N. et al. The role of scattering in quasi-ordered structures for terahertz imaging: local order can increase an image quality // IEEE Transactions on Terahertz Science and Technology 2018. V. 8, No. 4, P. 403-409.

8. Tuchin, V. V. Tissue Optics: Light Scattering Methods and Instruments for Medical Diagnostics 2015. 3rd ed. Bellingham.

9. Stamnes, K. et al. Numerically stable algorithm for discrete-ordinate-method radiative transfer in multiple scattering and emitting layered media // Applied Optics 1988. V. 27, No. 12, P. 2502-2509.

10. Waterman, $P$. Matrix formulation of electromagnetic scattering // Proc. IEEE 1965. V. 53, No. 8, P. 805-812.

11. Taylor, A. $H$. The measurement of diffuse reflection factors and a new absolute reflectometer, // J. Opt. Soc. Amer. 1920. V. 115, No. 19, P. 9-23.

12. Zaytsev, K. I. et al. Accuracy of sample material parameters reconstruction using terahertz pulsed spectroscopy // Journal of Applied Physics 2014. V. 115, No. 19, P. 193105.

13. Zaytsev, K. I. et al. Invariant embedding technique for medium permittivity profile reconstruction using terahertz time-domain spectroscopy // Optical Engineering 2013. V. 52, No. 6, P. 068203. 